\title{
BMJ Open Design and rationale of a randomised controlled trial comparing apixaban to phenprocoumon in patients with atrial fibrillation on chronic haemodialysis: the AXADIA-AFNET 8 study
}

Holger Reinecke, ${ }^{1}$ Sabine Jürgensmeyer, ${ }^{2}$ Christiane Engelbertz, ${ }^{1}$ Joachim Gerss, ${ }^{3}$ Paulus Kirchhof, ${ }^{2,4}$ Günter Breithardt, ${ }^{1}$ Rupert Bauersachs, ${ }^{5,6}$ Christoph Wanner ${ }^{7}$

To cite: Reinecke H, Jürgensmeyer S, Engelbertz C, et al. Design and rationale of a randomised controlled trial comparing apixaban to phenprocoumon in patients with atrial fibrillation on chronic haemodialysis: the AXADIAAFNET 8 study. BMJ Open 2018;8:e22690. doi:10.1136/ bmjopen-2018-022690

- Prepublication history and additional material for this paper are available online. To view these files, please visit the journal online (http://dx.doi. org/10.1136/bmjopen-2018022690).

Received 6 March 2018 Revised 30 May 2018 Accepted 25 July 2018
Check for updates

(C) Author(s) (or their employer(s)) 2018. Re-use permitted under CC BY-NC. No commercial re-use. See rights and permissions. Published by BMJ.

For numbered affiliations see end of article.

Correspondence to

Professor Holger Reinecke;

holger.reinecke@ukmuenster.de

\section{ABSTRACT}

Introduction Patients with end-stage kidney disease requiring maintenance haemodialysis treatment experience a dramatic cardiovascular morbidity and mortality. Due to the high atherosclerotic and arteriosclerotic burden and profound alterations in haemostasis, they frequently suffer and die from both thromboembolic and bleeding events. This is a particular concern in patients on haemodialysis with atrial fibrillation (AF). Controlled trials on the optimal anticoagulation in patients with $\mathrm{AF}$ on haemodialysis are not available. The randomised controlled phase IIIb AXADIA-AFNET 8 trial will evaluate the safety and efficacy of the factor $\mathrm{Xa}$ inhibitor apixaban in patients with $\mathrm{AF}$ requiring haemodialysis.

Methods and analysis A total of 222 patients will be randomised in an open-labelled, 1:1 design to receive either apixaban $2.5 \mathrm{mg}$ twice daily or dose-adjusted vitamin $\mathrm{K}$ antagonist therapy (target international normalised ratio 2.0-3.0). All patients will be treated and followed up for a minimum of 6 months up to a maximum of 24 months. The primary outcome is major or clinically relevant, non-major bleedings or death of any cause. Secondary outcomes include stroke, cardiovascular death and other thromboembolic events, thus exploring the efficacy of apixaban. The first patient was randomised in June 2017.

Ethics and dissemination The study protocol was approved by the Ethical Committee of the Landesaertzekammer, Westfalen-Lippe and the Medical Faculty of the University of Muenster, Muenster, Germany (reference number: 2016-598f-A). Written informed consent will be obtained from all patients prior to study participation, including their consent for long-term followup. AXADIA-AFNET 8 is an investigator-initiated trial. Sponsor is AFNET, Muenster, Germany. Study findings will be disseminated to Bristol-Myers Squibb, Munich, Germany, and Pfizer, Berlin, Germany, to the participating centres, at research conferences and in peer-reviewed journals.

Trial registration numbers NCT02933697,Pre-results.
Strengths and limitations of this study

- This randomised controlled phase IIlb trial will provide important information about the safety of apixaban compared with vitamin $\mathrm{K}$ antagonist in patients on haemodialysis and with non-valvular atrial fibrillation.

- The secondary outcome will evaluate the efficacy of apixaban compared with vitamin $\mathrm{K}$ antagonist in preventing thromboembolic events.

- A pharmacokinetic substudy enrolling 28 patients will investigate whether a reduced dosage of $2.5 \mathrm{mg}$ apixaban in haemodialysis patients results in similar plasma levels of apixaban as the recommended $5 \mathrm{mg}$ dose for patients without renal insufficiency, as preliminary findings suggest.

- This trial will provide evidence which type of anticoagulation shall be preferred in patients on haemodialysis and with non-valvular atrial fibrillation.

- The lack of a control arm without any oral anticoagulation is a limitation of the study design.

\section{INTRODUCTION}

More than 15 million people in the USA and more than 6 million people in Germany suffer from chronic kidney disease (CKD), with an expected increase in ageing populations with more comorbidities such as diabetes or hypertension. ${ }^{1-6}$ Approximately 120000 patients are alive in CKD stage G4 in Germany, and 85000 in stage G5D (end-stage kidney disease (ESKD) on peritoneal or haemodialysis). ${ }^{5}$ CKD is an important driver of cardiovascular mortality, ${ }^{4}$ illustrating the need for optimal cardiovascular protection in patients with CKD. Atrial fibrillation (AF) is common in CKD affecting ca. $10 \%-25 \%$ of patients with end-stage CKD. ${ }^{7-10}$

Patients with CKD are at high risk of thromboembolic events and bleedings. ${ }^{78}$ Concordantly, ischaemic and haemorrhagic stroke 
are typical and frequent complication in ESKD: data from the large US Renal Data System showed a stroke incidence of $15.1 \%$ in haemodialysis patients compared with $9.6 \%$ in other stages of CKD, and $2.6 \%$ in a control cohort without CKD; stroke-related 2-year mortalities of $74 \%, 55 \%$ and $28 \%$, respectively, have been reported from some analyses. ${ }^{11}$ Oral anticoagulation with vitamin $\mathrm{K}$ antagonists (VKAs) can prevent most thromboembolic events in patients with AF at risk of stroke. ${ }^{12}$ Non vitamin $\mathrm{K}$ antagonist oral anticoagulants (NOACs) have a similar effectiveness with lower risk of bleeding, particularly intracranial bleeds. ${ }^{13}$

The effectiveness and safety of VKA and NOACs in patients with non-valvular AF is well established, including in patients with mild or moderate CKD. ${ }^{12-14}$ Unfortunately, there is very little evidence on the safety and efficacy of NOACs in patients with AF and end-stage CKD.

Bleeding rates in CKD patients on dialysis treated with VKAs are high, ${ }^{15-19}$ illustrating the need for safe anticoagulation in these patients. ${ }^{20}$ In a retrospective analysis of the ARISTOTLE data, ${ }^{21}$ the reduction in major bleedings was greatest in patients in advanced CKD on apixaban compared with patients on VKA. Apixaban or other NOACs have not been evaluated in patients with AF on haemodialysis. To address this evidence gap, the 'Safety Study Assessing Oral Anticoagulation with Apixaban versus Vitamin K Antagonists in Patients with Atrial Fibrillation and End-Stage Kidney Disease (ESKD) on Chronic Hemodialysis Treatment' (AXADIA-AFNET 8) will compare the safety of apixaban to VKA therapy in CKD patients on dialysis.

\section{METHOD AND ANALYSIS}

\section{Primary and secondary objective}

The primary objective is to assess the safety of apixaban compared with VKA therapy (carried out using the local VKA phenprocoumon) in patients with AF and ESKD on maintenance haemodialysis treatment. The primary hypothesis of the study is that apixaban has a superior safety profile in patients with AF and ESKD on haemodialysis compared with phenprocoumon, while providing similar efficacy in reducing thromboembolic events. The secondary objective is to compare the efficacy of apixaban with VKA regarding prevention of thromboembolic events in patients with ESKD on haemodialysis and AF.

\section{Patients, study design and randomisation}

AXADIA-AFNET 8 is an investigator-initiated, prospective, parallel-group, multicentre trial conducted in 25-30 German study sites (online supplementary appendix A). Adult patients aged $\geq 18$ years with ESKD on maintenance haemodialysis treatment three times a week and an indication for oral anticoagulation due to non-valvular AF will be centrally and electronically randomised into two treatment arms: apixaban or phenprocoumon (1:1 allocation). As phenprocoumon needs constant international normalised ratio (INR) control, the study will be performed with an open-label administration of the study drugs. The randomisation will be stratified by two conditions: First, patients with a previous thromboembolism including any type of ischaemic stroke that meet the above criteria can be included after $>3$ months if they are not severely handicapped as indicated by a value of 0 or 1 on the modified Rankin scale ${ }^{22}$ and will be stratified to assure equal distribution within the groups. For further details on inclusion and exclusion criteria, see Box 1 .

Second, patients actually on phenprocoumon therapy can also be randomised; however, their proportion will be limited to a maximum $50 \%$ of the entire cohort to ensure that AXADIA-AFNET 8 also provides safety information in anticoagulation-naive patients. This condition will also be concerned as stratification in the randomisation procedure. In overview, patients' recruitment and allocation is displayed in figure 1 in accordance to the Consolidated Standards of Reporting Trials (CONSORT) standard.

\section{STUDY PROCEDURES}

\section{Treatment with apixaban}

Patients randomised to apixaban will be treated with apixaban $2.5 \mathrm{mg}$ twice a day. Apixaban will be provided to the study sites by Bristol-Myers Squibb in specific labelled bottles of 200 tablets. Apixaban was chosen as its mode of excretion is mainly via the bile, theoretically enabling stable plasma levels independent of haemodialysis. In addition, a subanalysis of the effect of CKD in the ARISTOTLE trial suggested that the relative bleeding risk of apixaban compared with VKA was lower in patients with advanced CKD (estimated glomerular filtration rate (eGFR) $25-50 \mathrm{~mL} / \mathrm{min}$ ). ${ }^{21}$ The study drug will be dispensed to the patient at the study site. The patient will be instructed to take the study drug with an approximately 12 hours gap apart. The compliance will be controlled by pill count and treatment interruptions will be documented and tracked centrally in the e-trial management system.

\section{Treatment with phenprocoumon}

Patients randomised to VKA will be treated with phenprocoumon, the locally used VKA in Germany, with a target INR of 2.0-3.0. Patients will receive a prescription for phenprocoumon at the study site on the day of randomisation. According to measured INR and the summary of product characteristics (SmPC), the investigator will recommend an individually adjusted dose to the patient. Weekly INR controls will be carried out to ensure the compliance to the target INR.

The protocol provides detailed recommendations for anticoagulation during haemodialysis (online supplementary appendix B).

All subjects will be treated with the study drug for at least 6 months. Subjects who are included early during the trial will stay under treatment in the study until the last subject has completed the treatment period of 6 


\section{Box 1 Inclusion and exclusion criteria}

\section{Inclusion criteria}

- End-stage kidney disease with chronic haemodialysis treatment three times per week (with about 4 hours per dialysis).

- Chronic (ie, repeated) paroxysmal, persistent or permanent AF documented by standard or Holter ECG on at least two separate days before (or apart from) haemodialysis procedures.

- Increased risk of stroke or systemic embolism identified by a $\mathrm{CHA}_{2} \mathrm{DS}_{2}$-VASc score of $\geq 2$ as an indication for oral anticoagulation.

- Patients with a previous ischaemic stroke that meet the above criteria can be included after $>3$ months if not severely handicapped (modified Rankin scale 0 or 1 of $6^{22}$ ).

- Males and females, aged 18 or older.

- Signed written informed consent.

\section{Exclusion criteria at baseline}

$\checkmark \mathrm{AF}$ or atrial flutter due to reversible causes (eg, thyrotoxicosis, pericarditis).

- Patients with a new onset of haemodialysis within the last three months.

- Clinically significant (moderate or severe) aortic and mitral stenosis.

- Conditions other than $\mathrm{AF}$ that require chronic anticoagulation (eg, a prosthetic mechanical heart valve).

- Active infective endocarditis.

- Any planned interventional or surgical AF or atrial flutter ablation procedure.

- Any active bleeding.

- A serious bleeding event in the previous sixmonths before screening.

- Inadequately controlled (HbA1c levels $>8.5 \%$ ) or untreated diabetes.

- History of malignant neoplasms at high risk of current bleeding (see summary of product characteristics of study drugs).

- Known indication for treatment with NSAIDs (ASA up to $100 \mathrm{mg}$ per day is allowed).

- Impaired liver function, for example, caused by active infection with HIV, HBV or HCV, hepatitis or other liver damage.

- Any type of stroke within 3 months prior to baseline.

- Valvular heart disease requiring surgery.

- A high risk of bleeding (eg, active peptic ulcer disease, a platelet count of $<100000 / \mathrm{mm}^{3}$ or haemoglobin level of $<8 \mathrm{~g} / \mathrm{dL}$ ).

- Documented haemorrhagic tendencies or blood dyscrasias.

- Current alcohol or drug abuse.

- Life expectancy of $<1$ year.

- Indication for dual platelet inhibition at baseline (ASA $\leq 100 \mathrm{mg} /$ day is allowed; clopidogrel is at baseline excluded at any dose, during trial allowed for up to 30 days).

- Any disease or circumstances on account of which the subject should not participate in the study in the opinion of the investigator.

- Psychiatric condition that might limit the participation in the study and/or that lead to the assumption that the ability to completely understand the consequences of consent is missing.

- Pregnant or breastfeeding women.

- Documented intolerance to one or both active substances of the study drugs.

AF, atrial fibrillation; ASA, acetylsalicylic acid; $\mathrm{CHA}_{2} \mathrm{DS}_{2}$-VASc score, congestive heart failure/LV dysfunction, hypertension, age $\geq 75$ years, diabetes, previous stroke/transischaemic attack/thromboembolism, vascular disease, age 65-74, sex category (female); HBV, hepatitis B virus; HCV, hepatitis C virus; NSAIDs, non-steroidal anti-inflammatory drugs. months. This will probably lead to a study participation of about 24 months for the first subjects enrolled (figure 2).

\section{Timelines and follow-up}

Screening period (up to 7 days before baseline visit)

In presence of the study staff, all patients must give full written informed consent during the screening period (online supplementary appendix C). Insurance information covering any harm resulting from the study drug is handed out to the participant. Inclusion and exclusion criteria will be checked. Demographic and anthropometric data, renal status, concomitant diseases and medication, and data on haemodialysis will be documented. Blood count, INR evaluation, ECG recording and risk stratification will be performed.

\section{Baseline visit}

Adherence to inclusion and exclusion criteria will be checked. Data on haemodialysis and concomitant medication will be documented. If applicable, blood parameters, thromboembolic and bleeding events, adverse events (AEs) and events of special interest will be recorded. Patients will be randomised. EQ-5D questionnaire will be completed by the patient. For subjects randomised for phenprocoumon, INR evaluation will be performed.

\section{Follow-up visits}

During the monthly follow-up visits, data on haemodialysis and INR evaluation (for subjects on phenprocoumon) will be documented. If applicable, changes in concomitant medication, blood parameters, thromboembolic and bleeding events, AEs and events of special interest will be documented. EQ-5D questionnaire will be completed on visits 6,12 and 18 by the patient. Adherence to study drug will be performed on a quarterly basis.

\section{End of treatment}

Data on haemodialysis, concomitant medication, concomitant diseases and adherence to study drug will be documented. ECG recording will be performed. EQ-5D questionnaire will be completed by the patient. For subjects on phenprocoumon, INR will be measured. If applicable, thromboembolic and bleeding events, AEs and events of special interest will be recorded.

\section{End of study (30 days after end of treatment)}

Data on concomitant medication, blood parameters, thromboembolic and/or bleeding events, AEs and events of special interest will be documented.

\section{AE reporting}

An $\mathrm{AE}$ is defined as any untoward medical occurrence or worsening of a pre-existing medical condition in the patient administered the study drug and that does not necessarily have a causal relationship with the study drug (ie, apixaban or phenprocoumon). All AEs, regardless of related or not related to the study drug, which occur from screening to end of study will be collected and 


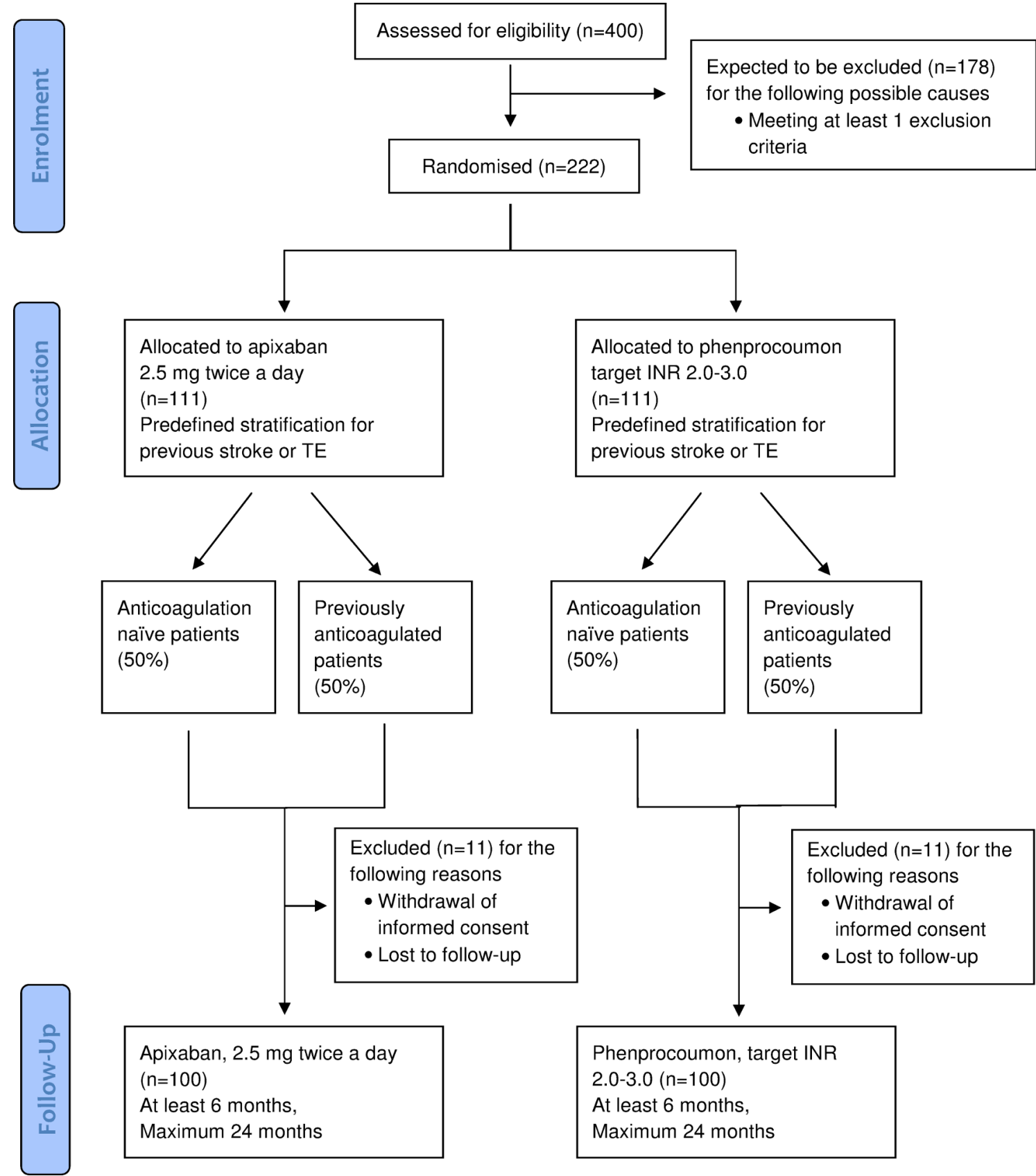

Figure 1 Patients' enrolment and allocation in accordance to the Consolidated Standards of Reporting Trials (CONSORT) stand is displayed. Of note, two stratifications are implemented in the randomisation procedure: first, for patients who suffered from a previous stroke or systemic thromboembolic (TE) event which must have occurred at least 3 months before enrolment without residual limitations (only value of 0 or 1 on the modified Rankin scale). Second, for patients who were already assigned to an oral anticoagulation whose proportion will be limited to $50 \%$ of the entire study cohort. INR, international normalised ratio.

seriousness, severity and causality will be assessed by the investigator. All AEs will be reported in detail in the electronic case report form (eCRF).

\section{Primary, secondary and other outcomes}

Primary outcome is a composite of major and clinically relevant, non-major bleeding in accordance to the International Society of Thrombosis and Hemostasis (ISTH) consensus $^{23}$ as well as shunt/fistula bleedings in dialysis patients on anticoagulation. The detailed list of all safety end points is given in Box 2.
Secondary outcomes include thromboembolic events. Details of the secondary outcomes and other outcomes are given in Box 2.

\section{Study discontinuation}

In accordance to the SmPC of apixaban, ${ }^{24}$ the following medications are prohibited throughout the whole trial and will lead to study discontinuation: Strong inhibitors of both cytochrome P450 3A4 and P-glycoprotein. Furthermore, regular intake of non-steroidal anti-inflammatory drugs (NSAIDs) or cyclo-oxygenase-2 inhibitors, except 


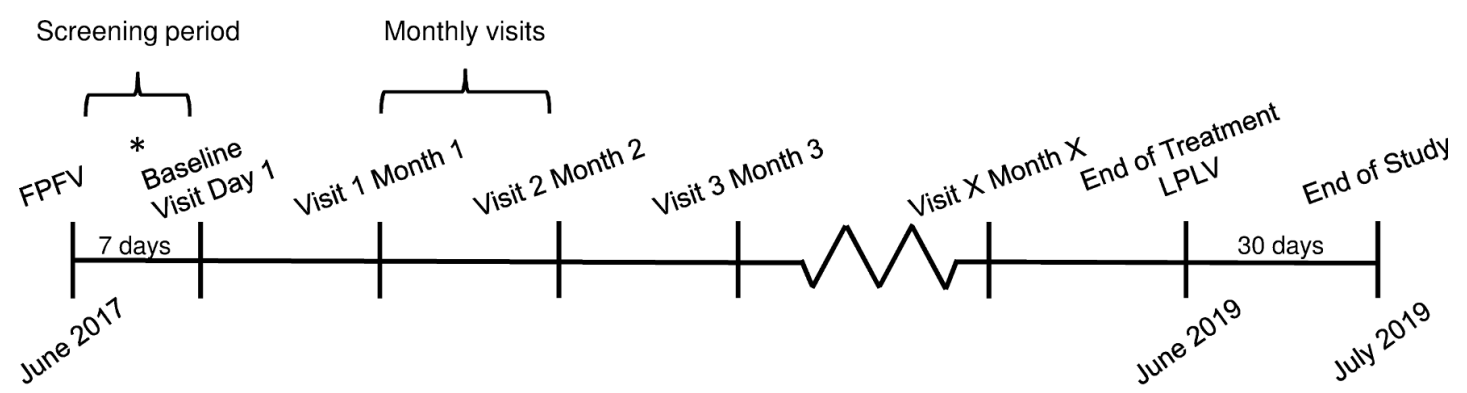

\footnotetext{
*: Randomisation

FPFV: first patient first visit

LPLV: last patient last visit
}

Figure 2 Timelines and regular visits during the study period are presented. After screening visit, two qualifying ECGs showing atrial fibrillation must be sent to a central appraisal and, if all other inclusion and exclusion criteria match, randomisation and inclusion can be performed within 7 days. Afterwards, monthly visits during the regular dialysis sessions will have to be documented. When the last recruited patient has been on treatment for 6 months, the study will be terminated (end of treatment). All patients will be followed up for another 30 days after which the study will be completed (end of study).

for acetylsalicylic acid (ASA) up to $100 \mathrm{mg}$ /day, lead also to study discontinuation.

Other reasons for discontinuation are elective surgical interventions which demands interruption of study drug intake for >3weeks; planned kidney transplantation within the next three days; pregnancy; and any $\mathrm{AE}$ or clinical relevant change in blood parameters because of which the responsible physician does not consider a study drug continuation as beneficial for the patient.

All patients who prematurely discontinue the study drug treatment are asked to perform the end of treatment visit.

\section{Data management and auditing}

An interactive web response system will be used to allocate patients to the two treatment groups. Study staff will record patients' data on the eCRF. All study-related information about participants will be stored securely and kept strictly confidential. All study sites will be supervised by a monitor throughout the entire study duration. One visit takes place before enrolment of the first patient to clarify all prerequisites and assure the medical and formal instruction of investigators and the knowledge to the protocol. On-site monitoring takes place on a regular basis and includes review of protocol compliance, check of the complete and correct entry into the eCRF, and correct handling and storage of the study drug.

\section{Patient and public involvement}

Neither public nor patients were involved in the design or conduct of the study. Study results will be disseminated to the patients by a circular letter.

\section{Pharmacokinetic substudy}

The dosing of apixaban with $2.5 \mathrm{mg}$ twice daily was chosen from pharmacokinetic modelling from plasma levels of some individuals with dialysis-dependent renal failure. Moreover, it is based on the currently recommended dose for apixaban in patients with CKD stage G4 according to the European label. ${ }^{24}$ Furthermore, results from a small pivotal study were published indicating that a dosage of $2.5 \mathrm{mg}$ apixaban twice daily in dialysis-dependent patients will result in similar plasma levels as the higher dosage of $5 \mathrm{mg}$ twice daily in healthy individuals. ${ }^{25}$ To confirm these preliminary findings, a pharmacokinetic substudy will be conducted in this trial with 28 patients randomised to apixaban to determine plasma serum levels of apixaban prior to and after haemodialysis, and also after a short (2 days) and a long (3 days) dialysis interval (figure 3). The study staff will obtain written consent from patients willing to participate in the substudy. In these patients, two blood samples will be collected, one sample before and one after haemodialysis. Apixaban levels will be measured at a central laboratory in Richmond, Virginia, USA.

\section{Sample size calculations}

One recent publication reported bleeding rates in a similar (but not identical) setting in haemodialysis patients treated with dabigatran and rivaroxaban, ASA and VKAs. ${ }^{26}$ Based on these data and using similar end point definitions especially for bleedings, it was assumed for the AXADIA-AFNET 8 study setting that apixaban would have comparable bleeding rates as ASA in dialysis patients. The latter assumption is supported by the AVERROES trial ${ }^{27}$ in which apixaban and ASA were compared in a cohort of non-dialysis-depended patients who were not considered suitable for oral anticoagulation due to a high bleeding risk: in that trial including patients with a comparable high risk as here, the rates of major and clinically relevant, non-major bleedings were similar in the apixaban group and the ASA group. Based on these data, we expect an hazard rate (HR) for the combination of major and clinically relevant non-major bleedings of 0.0789 per months for apixaban and 0.131 for phenprocoumon. According to these figures, a total sample size of 166 patients will be sufficient to demonstrate superiority of apixaban treatment with $80 \%$ power with respect to the primary end point, and also with respect to clinically 


\section{Box 2 End points}

Primary outcome: Composite of major and clinically relevant, non-major bleeding events and death of any cause

Major bleeding events as defined by ISTH ${ }^{23}$ and complemented by other serious bleeding events:

- Fatal bleeding.

- Bleeding that is symptomatic and occurs in a critical area or organ, such as intracranial, intraspinal, intraocular, retroperitoneal, pericardial, in a non-operated joint or intramuscular with compartment syndrome.

- Bleeding causing a fall in haemoglobin level of $20 \mathrm{~g} / \mathrm{L}(1.24 \mathrm{mmol} / \mathrm{L})$ or more.

- Bleeding leading to transfusion of two or more units of whole blood or red cells, with temporal association within 24-48hours to the bleeding.

- Bleeding that requires an operation or endoscopic intervention (arthroscopic, endovascular or a haemarthrosis).

Clinically relevant, non-major bleeding (according to ISTH consensus, ${ }^{23}$ complemented by relevant bleeding events in dialysis patients):

- Bleeding resulting in hospitalisation or prolonged hospitalisation.

- Bleeding requiring medical or surgical treatment by a physician.

- Bleeding leading to a modification of the given anticoagulant therapy.

- Gastrointestinal bleeding proven by endoscopy or surgery.

- Shunt-induced/catheter-induced bleeding.

- Bleeding between dialysis sessions.

- Prolonged bleeding requiring compression for $>30$ min after dialysis needle removal.

Efficacy events

Myocardial infarction.

- Ischaemic stroke.

- All-cause death.

- Cardiovascular death.

- Deep vein thrombosis and/or pulmonary embolism.

Composite end point

Myocardial infarction.

- Ischaemic stroke.

- All-cause death.

Deep vein thrombosis and/or pulmonary embolism.

Events of special interest

- Dialysis shunt thrombosis.

- Clotting of dialysis membrane.

Pharmacokinetics (substudy in $\mathbf{2 8}$ apixaban patients)

- Apixaban plasma level prior and after haemodialysis, see figure 3.

ISTH, International Society on Thrombosis and Haemostasis.

relevant, non-major bleedings only. Including a $20 \%$ security margin and about $10 \%$ drop-outs, AXADIA-AFNET 8 will randomise 222 patients (figure 1).

\section{Statistics}

The full analysis set will include all randomised patients with and without any kind of protocol violations, applying the intention-to-treat-principle. In the per-protocol population, patients with relevant protocol violations will be excluded. The safety set will consist of all patients who were randomised and received at least one dose of the study drug, applying the as-treated principle.

Continuous variables will be presented group-wise by means $\pm \mathrm{SD}$ and tested by t-tests for independent samples.
Skewed data will be transformed towards normality or tested by non-parametric $\mathrm{U}$ tests. Categorical variables will be presented by counts and percentages and compared by $\chi^{2}$ tests or Fisher's exact tests as appropriate.

In the primary statistical analysis, the treatments will be compared with respect to the primary safety end point time to first occurrence of a major or clinically relevant, non-major bleeding or death of any cause. Let HR denote the corresponding HR of the apixaban treatment versus VKA (phenprocoumon) treatment. Two statistical null hypotheses will be tested:

$\mathrm{H}_{0}^{\text {Superiority }}$ : $\mathrm{HR} \geq 1$ versus $\mathrm{H}_{1}^{\text {Superiority }}$ : $\mathrm{HR}<1$ (proof of superiority of apixaban)

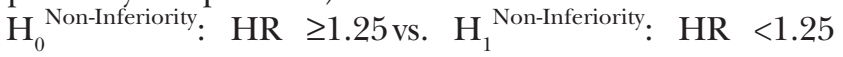
(proof of non-inferiority of apixaban)

The multiple one-sided significance level is set to $\alpha=2.5 \%$. A sequentially rejective multiple test procedure will be applied that controls the familywise error rate in the strong sense at prespecified significance level $\alpha$.

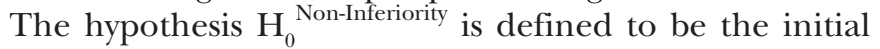
hypothesis and is assigned the local significance level $\alpha\left(\mathrm{H}_{0}^{\text {Non-Inferiority }}\right)=2.5 \%$. If the initial hypothesis $\mathrm{H}_{0}^{\text {Non-Infe- }}$ riority is rejected, subsequently the hypothesis $\mathrm{H}_{0}^{\text {Superiority is }}$ tested on local significance level $2.5 \%$. The primary statistical analysis provides confirmatory statistical evidence.

The superiority test will be performed in the full analysis set that consists of all randomised patients, including patients with any kind of protocol violations, applying the intention-to-treat principle. The non-inferiority test will additionally be performed in the per-protocol population, excluding patients with relevant protocol violations. Statistically significant non-inferiority will be claimed only if the result is supported both in the intention-to-treat analysis and the per-protocol analysis.

The primary analysis will be up to the first occurrence of the end point. Beyond the above primary statistical analysis of the primary end point, an extended analysis of the primary end point will additionally take recurrent events into regard and will be performed by fitting an Anderson-Gill model with robust estimation of coefficients and variance terms to the data. From all models, HRs and two-sided $95 \%$ confidence limits will be calculated and discussed whether safety risks can be derived based on the results. Statistical analyses of the primary end point will be repeated in the safety population that consists of all patients who received at least one dose of the study drug, applying the as-treated principle.

The secondary end points, both events of special interest and the composite end point (Box 2) are the efficacy end points and will be analysed on an intention-to-treat-basis by descriptive statistics only due to the small sample size which will probably not be sufficiently large enough to demonstrate superiority. Adjusted Cox proportional hazard models, Anderson-Gill models and the described sensitivity analyses will be performed as described above. No adjustment for multiplicity will be provided as the efficacy analysis is secondary behind the primary safety analysis. 


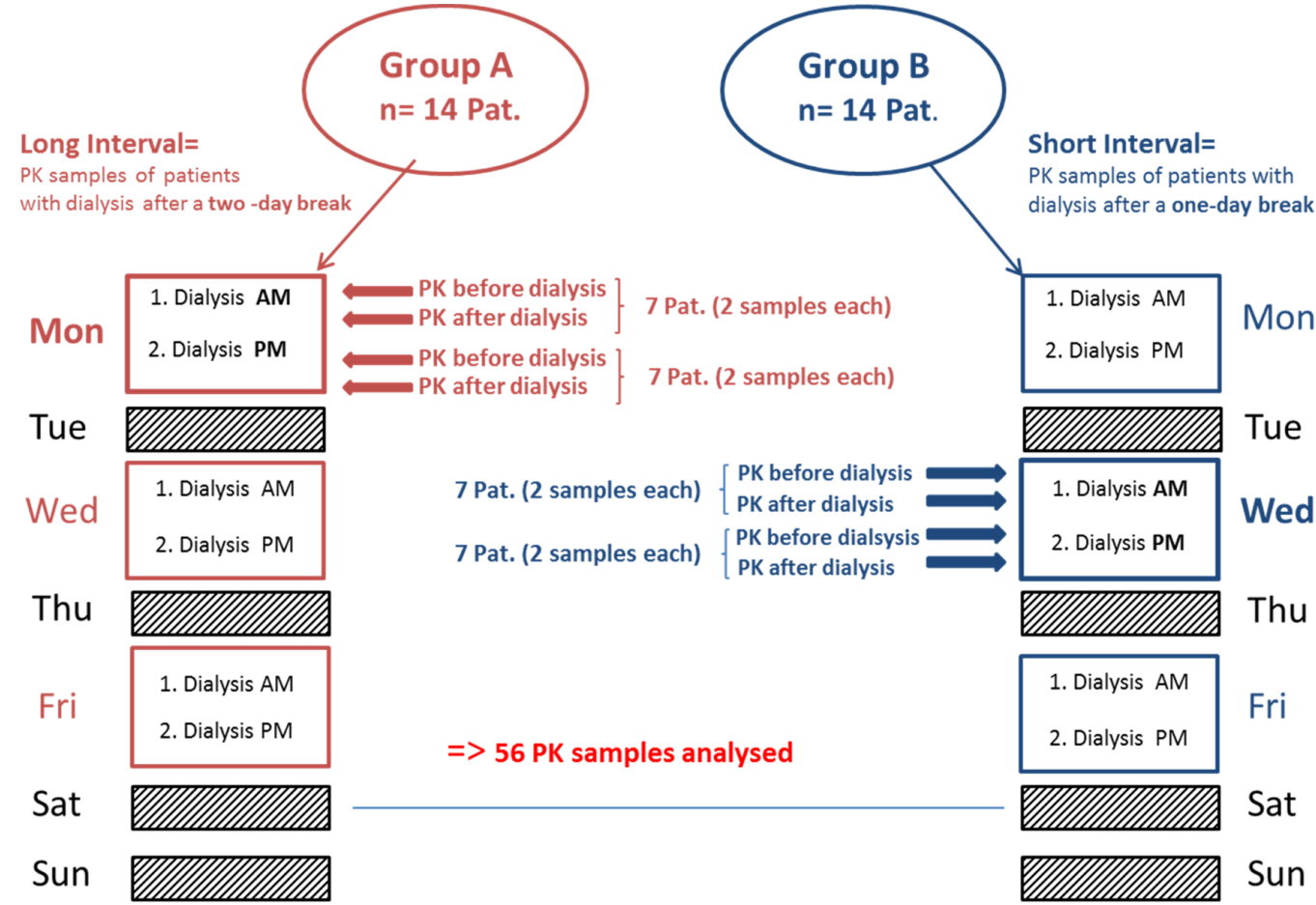

Figure 3 The design of a pharmacokinetic (PK) substudy is given here. In total, 28 patients who must give additional written informed consent will be examined regarding their apixaban levels before and after dialysis. Of these, apixaban levels will be assessed in 14 patients after the 3-day-long dialysis-free interval, and in another 14 patients after the short 2-day dialysis-free interval. Since patients on haemodialysis are regularly treated in two shifts (AM and PM), the 14 patients will be splitted again and apixaban levels will be assessed in 7 patients of the AM shift and another 7 patients of the PM shift. This might be of interest since patients will be instructed to take their apixaban between 07:00 to 08:00 AM and PM, independent from the start of their dialysis shifts, so plasma levels may vary between the groups.

The Data and Safety Monitoring Board will adjudicate the following safety and efficacy end points: major bleeding, clinically relevant, non-major bleeding, myocardial infarction, ischaemic stroke, all-cause death, cardiovascular death and deep vein thrombosis and/or pulmonary embolism (Box 2).

\section{Ethics and dissemination}

AXADIA-AFNET 8 is an investigator-initiated trial which is conducted by the Kompetenznetz Vorhofflimmern e.V. (Atrial Fibrillation NETwork, AFNET), Muenster, Germany, as responsible sponsor. The study is financed by Bristol-Myers Squibb (BMS, Munich, Germany) and Pfizer (Berlin, Germany) as specified in the supplement (online supplementary appendix D).

AXADIA-AFNET 8 is registered at http://www.clinicaltrials.gov (NCT02933697, registration date 14 October 2016; pre-results) and at EudraCT (2015-005503-84).

The study protocol (CV185-435, V.1.2, 18 August 2017) was approved by the Ethical Committee of the Landesaerztekammer (Medical Association) Westfalen-Lippe and the Medical Faculty of the University of Muenster, Muenster, Germany (reference number: 2016-598f-A) as lead ethics committee and by ethics committees at all participating sites. Protocol amendments will be communicated to the study sites by the sponsor.

The design, conduct and analysis of the study as well as decisions on publication of study results will be led by a steering committee (online supplementary appendix E). The steering committee has full access to the data set. A Data and Safety Monitoring Board will supervise this study and will be blinded to treatment groups (online supplementary appendix F). All safety and efficacy end points will be evaluated by an independent Endpoint Assessment Committee blinded to treatment group, eCRF, e-trial system, data capture and monitoring (online supplementary appendix G). The trial sponsor has no role in collection, analysis and interpretation of data, or decision to submit results.

Study findings will be disseminated to Bristol-Myers Squibb, Munich, Germany, and Pfizer, Berlin, Germany, to the participating centres, at research conferences, and in peer-reviewed journals.

\section{DISCUSSION}

Patients with CKD are well known to suffer from a high overall morbidity and mortality which is mainly driven by cardiovascular causes. This concerns especially patients in the advanced CKD stages G3 to G5 as demonstrated impressively by estimated 2.2 million deaths worldwide which are attributed only to patients in these stages. ${ }^{4}$ These deleterious effects are caused, on the one hand, by the underlying diseases leading to kidney disease, on the other hand, by alterations induced by CKD itself, which lead to rapid progress in atherosclerosis and 
arteriosclerosis but also to profound changes in haemostasis. Apart from these disease-related origins, the poor prognosis of patients with CKD-and especially those with ESKD—is, moreover, also attributed to a 'therapeutic nihilism' of many treating physicians: many AF patients with advanced CKD do not receive medications or treatments that have been proven or are at least considered to be beneficial in the general population. ${ }^{28}$

\section{Oral anticoagulation in advanced kidney disease}

This therapeutic nihilism is also of relevance in an ongoing, very controversial-in part highly emotionaldebate about the value of oral anticoagulation in ESKD. Some of the protagonists accuse the others to harm the patients by administering a dangerous VKA, while their opponents point out that withholding the oral anticoagulation is not justified and actually harms the patients. In fact, currently no one has reliable and adequate data: while especially the large-sized trials with non-VKA oral anticoagulants have shown that oral anticoagulation is effective and safe even down to an eGFR of 25 or $30 \mathrm{~mL} /$ min, ${ }^{29-32}$ below this threshold the available data are markedly limited regarding both quantity and quality.

Numerous small and retrospective studies have tried to assess the value of VKA in haemodialysis as summarised previously. ${ }^{19} 33$ However, all retrospective studies suffer from a significant selection bias of the patients treated with anticoagulation. ${ }^{7}$ A meta-analysis of 11 observational studies with $25407 \mathrm{AF}$ patients with EKSD found warfarin to significantly lower stroke rate but to have no effect on mortality. ${ }^{34}$

Larger, unbiased observational information exists: in an analysis of the hospital database of Denmark with $>130000 \mathrm{AF}$ patients of whom 3587 (2.7\%) had non-endstage CKD and $901(0.7 \%)$ required renal replacement therapy at the time of inclusion, ${ }^{35}$ warfarin treatment was associated with a significantly decreased risk of stroke or systemic thromboembolism in patients with CKD and ESKD. VKA monotherapy and combination of VKA and ASA were associated with an increased risk of bleeding. Platelet inhibitors such as ASA did not reduce thromboembolic events but were associated with a high rate of adverse effects as also shown by others. ${ }^{35} 36$ Therefore, platelet inhibition does not seem to be a viable treatment in patients with AF and ESKD. A more recent report on an updated version of the same data set included meanwhile 150000 patients ${ }^{37}$ with an increased risk of stroke and systemic embolism $\left(\mathrm{CHA}_{2} \mathrm{DS}_{2}-\mathrm{VASc}\right.$ score $\left.\geq 2\right)$; their data showed that VKA therapy was significantly associated with lower all-cause mortality in patients with CKD (HR $0.64,95 \%$ CI 0.60 to 0.69 ) and in those with ESKD (HR $0.85,95 \%$ CI 0.72 to 0.99 ).

Another very recent publication from the same group has shown that time in therapeutic range (TTR) is of special importance in patients with reduced eGFR. ${ }^{38}$ This aspect of TTR is included in AXADIA and the INR will be assessed and documented thoroughly.
All of these analyses do yet not allow an evidence-based decision how to treat haemodialysis patients to reduce their immense risk of stroke, systemic and venous thromboembolic events, bleedings and cardiovascular death. A recently started trial in the USA (RENAL-AF, clinicaltrial.gov identifier NCT02942407) comparing apixaban ( $5 \mathrm{mg}$ twice daily) with warfarin (INR 2-3) in patients with AF and ESKD on haemodialysis will add knowledge regarding safety and efficacy of apixaban in this critically ill patients' cohort.

\section{Calciphylaxis with VKAs}

Patients on maintenance haemodialysis treatment are often vitamin K-deficient which may be linked with advanced arterial calcifications due to a number of preclinical and few clinical studies. ${ }^{39-42}$ However, a very recent clinical trial failed to demonstrate any negative impact of warfarin on calcification, ${ }^{43}$ while also one older but large observational study from the US renal data system could not find any difference in overall survival between patients with and without warfarin. ${ }^{44}$ Although no explicit substudy regarding vascular calcifications is planned within AXADIA, major cardiovascular events due to calcifications are included in the efficacy end points of the trial and will be compared with patients receiving VKA and those under apixaban.

\section{Limitations}

With regard to the fact that this is an investigator-initiated trial, financial resources are spare and had to be applied thoroughly. Therefore, the number of patients which will be included to determine a significant difference regarding the safety end points is considered to be sufficient. A security margin of $20 \%$ should compensate the complete lack of any previous data in this field. Nevertheless, a larger cohort would have been desirable in order to identify differences concerning the efficacy end points.

The trial is adequately designed and powered to answer the question whether apixaban is non-inferior or possibly even superior to phenprocoumon regarding safety and maybe also regarding efficacy. However, since there is no control arm without any anticoagulation regime, the trial will provide evidence which type of anticoagulation to prefer if indicated (and if intended by the treating physician) - but not, whether any oral anticoagulation should be given at all in haemodialysis-dependent patients with AF.

Fistula bleeding events may be induced either by puncturing for dialysis procedure or by anticoagulation. No differentiation will be made with regard to the underlying cause of this outcome measure. Therefore, bleeding events may be overestimated. But due to the randomised design of the trial, fistula bleedings are expected to occur with equal distribution in both trial arms.

Finally, the trial is designed as an open-label trial due to required regular dose adjustments for phenprocoumon based on INR measurements. 


\section{CONCLUSION}

The AXADIA trial will belong to the first randomised trials to compare two different regimens of anticoagulation in patients on maintenance haemodialysis. It will clarify if the novel direct factor Xa antagonist apixaban will be comparable or even be superior to a standard therapy with the VKA phenprocoumon in patients with paroxysmal, persistent or permanent AF. Finally, the AXADIA trial is a pilot study whose results shall provide the basis for a larger outcome study.

\section{Author affiliations}

${ }^{1}$ Dept. of Cardiology I - Coronary and Peripheral Vascular Disease, Heart Failure, University Hospital Muenster, Cardiol, Muenster, Germany

${ }^{2}$ Atrial Fibrillation NETwork, Muenster, Germany

${ }^{3}$ Institute of Biostatistics and Clinical Research, University of Muenster, Muenster, Germany

${ }^{4}$ Institute of Cardiovascular Sciences, University of Birmingham, and SWBH and UHB NHS Trusts, Birmingham, UK

${ }^{5}$ Department of Vascular Medicine, Klinikum Darmstadt, Darmstadt, Germany ${ }^{6}$ Center for Thrombosis and Hemostasis, University Medical Center of Johannes Gutenberg University of Mainz, Mainz, Germany

${ }^{7}$ Department of Medicine, Division of Nephrology, Wuerzburg University Clinic, Wuerzburg, Germany

Acknowledgements The authors are grateful to the Kuratorium für Heimdialyse (KfH), Neu-Isenburg, Germany, who support this trial by their members that act as participating centres. Furthermore, they thank Dr Eva Freisinger and Dr Katrin Gebauer for assistance and handling the SAE management.

Contributors HR, CW, RB, PK and GB conceived and designed the study; HR, $\mathrm{CW}, \mathrm{RB}, \mathrm{PK}, \mathrm{GB}$ and SJ contributed to protocol development; JG contributed to the statistical analysis; HR, CE and SJ participated in the writing of the manuscript; all authors critically reviewed the manuscript and approved the final version.

Funding This investigator-initiated trial is conducted by the Kompetenznetz Vorhofflimmern e.V. (Atrial Fibrillation NETwork), Mendelstr. 11, 48149 Muenster, Germany. The study is financed by Bristol-Myers Squibb (BMS), Munich, Germany, and Pfizer, Berlin, Germany.

Competing interests HR has received speaker honoraria from BMS, MedUpdate, NephroUpdate, and Pfizer. He has acted as a consultant for BMS, Pfizer and Pluristem receiving in part also financial compensations for this work. He has received research grants from the German Federal Ministry for Education and Research (BMBF). His division within the University Hospital of Muenster has taken or is still taking part in multicentre trials of BARD, Bayer, BIOTRONIK, and Pluristem receiving patient fees and financial compensation for these efforts. PK has received grants and non-financial support from the European Union, British Heart Foundation, Leducq Foundation, Medical Research Council (UK), and German Centre for Cardiovascular Research and from several drug and device companies active in atrial fibrillation, and has received honoraria from several such companies. He is listed as inventor on two patents held by the University of Birmingham (Atrial Fibrillation Therapy W0 2015140571, Markers for Atrial Fibrillation WO 2016012783). During preparation of this trial, GB has received speaker honoraria from BMS and Pfizer, he has been a member of the Scientific Advisory Boards for BMS and Pfizer, and Bayer Health Care. During his chairmanship of the Atrial Fibrillation NETwork, this institution has received funding for investigatorinitiated trials from various companies (for details, please consult http://www. kompetenznetz-vorhofflimmern.de/en/research). RB has received consulting / lecture fees from Bayer, Boehringer Ingelheim, Bristol-Myers Squibb, Daiichi Sankyo, and Pfizer. CW does not report conflicts of interest in respect to the present work. Outside this area of research he has received speaker honoraria from Amgen, Boehringer-Ingelheim, Genzyme-Sanofi and Shire.

Patient consent Not required.

Ethics approval Ethical Committee of the Landesaerztekammer (Medical Association) Westfalen-Lippe and the Medical Faculty of the University of Muenster, Muenster, Germany (reference number: 2016-598-f-A)

Provenance and peer review Not commissioned; externally peer reviewed.
Open access This is an open access article distributed in accordance with the Creative Commons Attribution Non Commercial (CC BY-NC 4.0) license, which permits others to distribute, remix, adapt, build upon this work non-commercially, and license their derivative works on different terms, provided the original work is properly cited, appropriate credit is given, any changes made indicated, and the use is non-commercial. See: http://creativecommons.org/licenses/by-nc/4.0/.

\section{REFERENCES}

1. Coresh J, Selvin E, Stevens LA, et al. Prevalence of chronic kidney disease in the United States. JAMA 2007;298:2038-47.

2. McCullough PA, Li S, Jurkovitz CT, et al. Chronic kidney disease, prevalence of premature cardiovascular disease, and relationship to short-term mortality. Am Heart J 2008;156:277-83.

3. Snyder JJ, Foley RN, Collins AJ. Prevalence of CKD in the United States: a sensitivity analysis using the National Health and Nutrition Examination Survey (NHANES) 1999-2004. Am J Kidney Dis 2009;53:218-28.

4. Thomas B, Matsushita K, Abate KH, et al. Global cardiovascular and renal outcomes of reduced GFR. J Am Soc Nephrol 2017;28:2167-79.

5. Reinecke H, Brandenburg V, Dominiak P, et al. Empfehlungen zur Diagnostik und Behandlung von Patienten mit koronarer Herzkrankheit und Niereninsuffizienz. Teil I: Pathophysiologie und Diagnostik. Clin Res Cardiol Supp/ 2006;1:8-30.

6. James MT, Hemmelgarn BR, Tonelli M. Early recognition and prevention of chronic kidney disease. Lancet 2010;375:1296-309.

7. Reinecke H, Brand E, Mesters R, et al. Dilemmas in the management of atrial fibrillation in chronic kidney disease. J Am Soc Nephrol 2009;20:705-11.

8. Marinigh R, Lane DA, Lip GY. Severe renal impairment and stroke prevention in atrial fibrillation: implications for thromboprophylaxis and bleeding risk. J Am Coll Cardiol 2011;57:1339-48.

9. Lau YC, Proietti M, Guiducci E, et al. Atrial fibrillation and thromboembolism in patients with chronic kidney disease. J Am Coll Cardiol 2016;68:1452-64.

10. Bansal N, Fan D, Hsu CY, et al. Incident atrial fibrillation and risk of end-stage renal disease in adults with chronic kidney disease. Circulation 2013;127:569-74.

11. U.S. Renal Data System, USRDS 2006 Annual Data Report: Atlas of End-Stage Renal Disease in the United States, National Institutes of Health, National Institute of Diabetes and Digestive and Kidney Diseases. Bethesda, MD, 2006

12. Kirchhof P, Benussi S, Kotecha D, et al. ESC Scientific Document Group. 2016 ESC Guidelines for the management of atrial fibrillation developed in collaboration with EACTS. Eur Heart $J$ 2016;37:2893-962

13. Ruff CT, Giugliano RP, Braunwald E, et al. Comparison of the efficacy and safety of new oral anticoagulants with warfarin in patients with atrial fibrillation: a meta-analysis of randomised trials. Lancet 2014;383:955-62.

14. January CT, Wann LS, Alpert JS, et al. American College of Cardiology/American Heart Association Task Force on Practice Guidelines. 2014 AHA/ACC/HRS guideline for the management of patients with atrial fibrillation: a report of the American College of Cardiology/American Heart Association Task Force on Practice Guidelines and the Heart Rhythm Society. J Am Coll Cardiol 2014;64:e1-76.

15. McCullough PA, Ball T, Cox KM, et al. Use of oral anticoagulation in the management of atrial fibrillation in patients with ESRD: pro. Clin J Am Soc Nephrol 2016;11:2079-84.

16. Keskar V, Sood MM. Use of oral anticoagulation in the management of atrial fibrillation in patients with ESRD: con. Clin J Am Soc Nephrol 2016;11:2085-92.

17. Bansal N. Use of oral anticoagulation for patients with esrd on hemodialysis with atrial fibrillation: Verdict 1. Clin J Am Soc Nephrol 2016:11:2093-4.

18. Herzog CA. Use of oral anticoagulation in the management of atrial fibrillation in patients with esrd: Verdict 2. Clin J Am Soc Nephrol 2016;11:2095-6.

19. Van Der Meersch H, De Bacquer D, De Vriese AS. Vitamin K antagonists for stroke prevention in hemodialysis patients with atrial fibrillation: A systematic review and meta-analysis. Am Heart $J$ 2017;184:37-46.

20. Reinecke H, Engelbertz C, Schäbitz WR. Preventing stroke in patients with chronic kidney disease and atrial fibrillation: benefit and risks of old and new oral anticoagulants. Stroke 2013;44:2935-41.

21. Hohnloser SH, Hijazi Z, Thomas L, et al. Efficacy of apixaban when compared with warfarin in relation to renal function in patients with 
atrial fibrillation: insights from the ARISTOTLE trial. Eur Heart $J$ 2012;33:2821-30.

22. Farrell B, Godwin J, Richards S, et al. The United Kingdom transient ischaemic attack (UK-TIA) aspirin trial: final results. J Neurol Neurosurg Psychiatry 1991;54:1044-54.

23. Schulman S, Kearon C. Subcommittee on Control of Anticoagulation of the Scientific and Standardization Committee of the International Society on Thrombosis and Haemostasis. Definition of major bleeding in clinical investigations of antihemostatic medicinal products in non-surgical patients. J Thromb Haemost 2005;3:692-4.

24. Summary of product characteristics. http://www.ema.europa.eu/ docs/en_GB/document_library/EPAR_-_Product_Information/human/ 002148/WC500107728.pdf. access date 28 Feb 2018

25. Mavrakanas TA, Samer CF, Nessim SJ, et al. Apixaban pharmacokinetics at steady state in hemodialysis patients. J Am Soc Nephrol 2017;28:2241-8.

26. Chan KE, Edelman ER, Wenger JB, et al. Dabigatran and rivaroxaban use in atrial fibrillation patients on hemodialysis. Circulation 2015;131:972-9.

27. Connolly SJ, Eikelboom J, Joyner C, et al. Apixaban in patients with atrial fibrillation. N Engl J Med 2011;364:806-17.

28. McCullough PA. Why is chronic kidney disease the "spoiler" for cardiovascular outcomes? J Am Coll Cardiol 2003;41:725-8.

29. Connolly SJ, Ezekowitz MD, Yusuf S, et al. Dabigatran versus warfarin in patients with atrial fibrillation. $N$ Engl J Med 2009;361:1139-51.

30. Fox KA, Piccini JP, Wojdyla D, et al. Prevention of stroke and systemic embolism with rivaroxaban compared with warfarin in patients with non-valvular atrial fibrillation and moderate renal impairment. Eur Heart $J$ 2011;32:2387-94.

31. Giugliano RP, Ruff CT, Braunwald E, et al. Edoxaban versus warfarin in patients with atrial fibrillation. N Engl J Med 2013;369:2093-104.

32. Granger $\mathrm{CB}$, Alexander JH, McMurray JJ, et al. Apixaban versus warfarin in patients with atrial fibrillation. $N$ Engl $J$ Med 2011;365:981-92.

33. Engelbertz $\mathrm{C}$, Reinecke $\mathrm{H}$. Atrial Fibrillation and Oral Anticoagulation in Chronic Kidney Disease. J Atr Fibrillation 2012;4:445-.
34. Liu G, Long M, Hu X, et al. Effectiveness and safety of warfarin in dialysis patients with atrial fibrillation: A meta-analysis of observational studies. Medicine 2015;94:e2233.

35. Olesen JB, Lip GY, Kamper AL, et al. Stroke and bleeding in atrial fibrillation with chronic kidney disease. N Engl J Med 2012;367:625-35.

36. Chan KE, Lazarus JM, Thadhani R, et al. Warfarin use associates with increased risk for stroke in hemodialysis patients with atrial fibrillation. J Am Soc Nephrol 2009;20:2223-33.

37. Bonde AN, Lip GY, Kamper AL, et al. Net clinical benefit of antithrombotic therapy in patients with atrial fibrillation and chronic kidney disease: a nationwide observational cohort study. J Am Coll Cardiol 2014;64:2471-82.

38. Bonde AN, Lip GY, Kamper AL, et al. Effect of reduced renal function on time in therapeutic range among anticoagulated atrial fibrillation patients. J Am Coll Cardiol 2017;69:752-3.

39. Schurgers LJ, Teunissen KJ, Hamulyák K, et al. Vitamin K-containing dietary supplements: comparison of synthetic vitamin K1 and nattoderived menaquinone-7. Blood 2007;109:3279-83.

40. Schlieper G, Westenfeld R, Krüger T, et al. Circulating nonphosphorylated carboxylated matrix gla protein predicts survival in ESRD. J Am Soc Nephrol 2011;22:387-95.

41. Weijs B, Blaauw $Y$, Rennenberg RJ, et al. Patients using vitamin $K$ antagonists show increased levels of coronary calcification: an observational study in low-risk atrial fibrillation patients. Eur Heart J 2011;32:2555-62.

42. Tantisattamo E, Han KH, O'Neill WC. Increased vascular calcification in patients receiving warfarin. Arterioscler Thromb Vasc Biol 2015;35:237-42.

43. Han KH, O'Neill WC. Increased peripheral arterial calcification in patients receiving warfarin. J Am Heart Assoc 2016;5:e002665.

44. Herzog CA, Ma JZ, Collins AJ. Long-term survival of dialysis patients in the United States with prosthetic heart valves: should ACC/AHA practice guidelines on valve selection be modified? Circulation 2002;105:1336-41. 